\title{
BORDISM OF MANIFOLDS WITH ORIENTED BOUNDARIES
}

\author{
G. E. MITCHELL
}

ABSTRACT. A bordism theory is defined for manifolds with oriented boundaries. The relation of this theory with the ordinary bordism theories is shown. These bordism classes are then characterized via characteristic numbers.

1. Introduction. In [10] Thom defined the oriented and unoriented bordism rings $\left(\Omega_{*}^{s o}\right.$ and $\Omega_{*}^{\circ}$, respectively) which classify oriented and unoriented compact manifolds without boundary. Conner and Floyd [4] and Atiyah [1] used Thom's ideas to develop generalized homology theories based on oriented and unoriented manifolds. In $\$ 2$ the geometric approach of Conner and Floyd is used to define a bordism theory for manifolds with oriented boundaries. Geometric constructions are given in $\$ 3$ which relate this bordism theory to the ordinary oriented theory. In $\$ 4$ a characteristic number classification of the bordism classes is given. Finally a remark is made concerning the homotopy interpretation of this bordism theory.

2. The $(O, S O)$-bordism theory. Consider the collection of pairs $\left(M^{n}, \partial M^{n}\right)$ consisting of a compact $n$-manifold $M^{n}$ with an oriented boundary $\partial M^{n} .\left(M^{n}, \partial M^{n}\right)$ is said to bord if there exists an $(n+1)$-manifold $W^{n+1}$ with boundary $\partial W^{n+1}$ ' such that:

(i) $M^{n} \subset \partial W^{n+1}$ as a regular submanifold, and

(ii) $\partial W^{n+1}-$ int $M^{n}$ is oriented in such a way that it induces the given orientation on $\partial M^{n}$. We say that $\left(M_{0}^{n}, \partial M_{0}^{n}\right)$ is bordant to $\left(M_{1}^{n}, \partial M_{1}^{n}\right)$ provided the disjoint union $\left(M_{0}^{n} \cup M_{1}^{n}, \partial M_{0}^{n} \cup-\partial M_{1}^{n}\right)$ bords. The standard methods [4], [6], [10] can be used to show that bordant is an equivalence relation in the class of all pairs $\left(M^{n}, \partial M^{n}\right)$. Let $\left[M^{n}, \partial M^{n}\right]$ denote the equivalence class of $\left(M^{n}, \partial M^{n}\right)$ and let $\Omega_{n}^{O, S O}$ denote the set of all such equivalence classes. As usual $\Omega_{n}^{O, S O}$ forms an abelian group under the operation of disjoint union and $\Omega_{*}^{S O}$ becomes an $\Omega_{*}^{S O}$-module under cartesian product.

Presented to the Society, June 30, 1969 under the title $(O, S O)$-bordism; received by the editors October 31, 1973 and, in revised form, December 12, 1973.

AMS (MOS) subject classifications (1970). Primary 57D75, 57D90; Secondary 57D20. 
The bordism groups $\Omega_{n}^{O, S O}$ are related to the ordinary oriented and unoriented bordism groups by the exact sequence

$$
\cdots \rightarrow \Omega_{n}^{S O} \rightarrow \Omega_{n}^{O} \stackrel{j}{\longrightarrow} \Omega_{n}^{O, S O} \stackrel{\partial}{\longrightarrow} \Omega_{n-1}^{S O} \stackrel{r}{\longrightarrow} \Omega_{n-1}^{O} \rightarrow \cdots
$$

The homomorphisms $i, \partial$ and $r$ are defined by

$$
j\left(\left[M^{n}\right]_{2}\right)=\left[M^{n}, \varnothing\right], \quad \partial\left(\left[M^{n}, \partial M^{n}\right]\right)=\left[\partial M^{n}\right] \quad \text { and } \quad r\left(\left[V^{n-1}\right]\right)=\left[V^{n-1}\right]_{2} .
$$

(The subscript 2 is used to denote an unoriented bordism class.) This exact sequence gives rise to the exact triangle of $\Omega_{*}^{S O}$-modules and $\Omega_{*}^{S O}$-homomorphisms

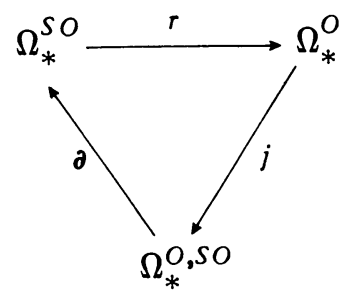

where $\partial$ has degree -1 .

Using the exact sequence (2.1) and facts from [7], [8], [10], [11] we see that

$$
\Omega_{n}^{O, S O} \cong \Omega_{n}^{O} / \mathrm{im} r \oplus 2 \Omega_{n-1}^{S O},
$$

where the elements from $\Omega_{n}^{O} / \mathrm{im} r$ are all of order 2 and $2 \Omega_{n-1}^{S O}$ is free abelian, Geometrically the elements of the free part of $\Omega_{n}^{O, S O}$ may be represented by pairs of the form $\left(I \times V^{n-1}, 1 \times V^{n-1} \cup O \times V^{n-1}\right)$, where $V^{n-1}$ is an oriented manifold and the elements of the torsion part can be represented by closed manifolds whose mod 2 bordism class contains no oriented manifolds.

An $(O, S O)$-pair is a pair $\left(M^{n}, B^{n-1}\right)$ consisting of an $n$-manifold $M^{n}$ with boundary $\partial M^{n}$ and an oriented regular submanifold $B^{n-1} \subset \partial M^{n}$. For a pair of spaces $(X, A)$ a singular $(O, S O)$-pair in $(X, A)$ is a pair $\left(M^{n}, B^{n-1} ; f\right)$ consisting of an $(O, S O)$-pair $\left(M^{n}, B^{n-1}\right)$ and a map $f: M^{n} \rightarrow X$ such that $f\left(\partial M^{n}-\right.$ int $\left.B^{n-1}\right) \subset A$. If $A=\varnothing$, it is assumed that $B^{n-1}=\partial M^{n}$.

A singular $(O, S O)$-pair $\left(M^{n}, B^{n-1} ; f\right)$ bords if there exists an $(O, S O)$ pair $\left(W^{n+1}, Q^{n}\right)$ and a map $F: W^{n+1} \rightarrow X$ such that

(i) $M^{n} \subset \partial W^{n+1}$ as a regular submanifold,

(ii) $B^{n-1} \subset \partial Q^{n}$ as a regular submanifold with the orientation on $\partial Q^{n}$ inducing the given orientation of $B^{n-1}$, 
(iii) $Q^{n} \cap M^{n}=B^{n-1}$ and $Q^{n} \cup M^{n}$ is a regular submanifold of $\partial W^{n+1}$, and

(iv) $F / M^{n}=f$, and $F\left(\partial W^{n+1}-\right.$ int $\left.\left(M^{n} \cup Q^{n}\right)\right) \subset A$.

Two singular $(O, S O)$-pairs $\left(M_{0}^{n}, B_{0}^{n-1} ; f_{0}\right)$ and $\left(M_{1}^{n}, B_{1}^{n-1} ; f_{1}\right)$ in $(X, A)$ are bordant if their disjoint union $\left(M_{0}^{n} \cup M_{1}^{n}, B_{0}^{n} \cup-B_{1}^{n-1} ; f_{0} \cup f_{1}\right)$ bords. Again the standard arguments can be used to show that this bordism relation is an equivalence relation. $\left[M^{n}, B^{n-1} ; f\right]$ will denote the class of $\left(M^{n}, B^{n-1} ; f\right)$, and $\Omega_{n}^{O, S O}(X, A)$ denotes the set of equivalence classes. $\Omega_{n}^{O, S O}(X, A)$ becomes an abelian group under the operation of disjoint union and $\Omega_{*}^{O, S O}(X, A)$ becomes an $\Omega_{*}^{S O}$-module under cartesian product.

Any map $h:(X, A) \rightarrow\left(X^{1}, A^{1}\right)$ induces a homomorphism $h_{*}: \Omega_{n}^{O, S O}(X, A)$ $\rightarrow \Omega_{n}^{O, S O}\left(X^{1}, A^{1}\right)$

defined by $h_{*}\left(\left[M^{n}, B^{n-1} ; f\right]\right)=\left[M^{n}, B^{n-1} ; b \circ f\right] . h_{*}$ is in fact an $\Omega_{*}^{S O}$-homomorphism.

Define the boundary homomorphism

$$
\partial: \Omega_{n}^{O, S O}(X, A) \rightarrow \Omega_{n-1}^{O, S O}(A)
$$

by $\partial\left(\left[M^{n}, B^{n-1}\right]\right)=\left[\partial M^{n}-\right.$ int $\left.\left(B^{n-1}\right), \partial B^{n-1} ; f^{1}\right]$, where $f^{1}$ is the restriction of $f$ to $\partial M^{n}-$ int $\left(B^{n-1}\right)$.

The techniques of [4] can now be used to prove

Theorem 1. $\left\{\Omega^{O, S O}(), h, \partial\right\}$ is a generalized homology theory on the category of pairs of spaces. The coefficient group $\Omega_{*}^{O, S O}(p t)=\Omega_{*}^{O, s O}$.

The bordism groups $\Omega_{n}^{O, S O}(X, A)$ are related to the ordinary oriented and unoriented bordism groups of the pair $(X, A)$ by the exact sequence

$$
\begin{aligned}
& \cdots \rightarrow \Omega_{n}^{S O}(X, A) \stackrel{i}{\longrightarrow} \Omega_{n}^{O}(X, A) \\
& \stackrel{j}{\longrightarrow} \Omega_{n}^{O, S O}(X, A) \stackrel{\partial}{\longrightarrow} \Omega_{n-1}^{S O}(X, A) \rightarrow \cdots,
\end{aligned}
$$

where $i, j$ and $\partial$ are given by

$$
i\left(\left[M^{n} ; f\right]\right)=\left[M^{n} ; f\right]_{2}, \quad j\left(\left[M^{n} ; f\right]_{2}\right)=\left[M^{n}, \varnothing ; f\right]
$$

and

$$
\partial\left(\left[M^{n}, B^{n-1} ; f\right]\right)=\left[B^{n-1} ; f / B^{n-1}\right] .
$$

This sequence can be arranged in an exact triangle 


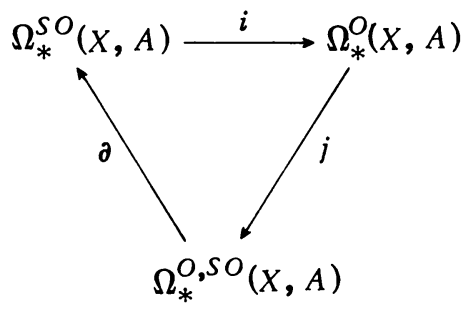

of $\Omega_{*}^{S O}$-modules and $\Omega_{*}^{S O}$-homomorphisms.

3. A geometric construction. In [9] Stong constructed an isomorphism

$$
\Delta: \Omega_{n}^{O, S O} \cong \Omega_{n-1}^{S O}(R P(\infty))
$$

Since $\Omega_{n-1}^{S O}(R P(\infty)) \cong \Omega_{n-1}^{S O} \oplus \Omega_{n-2}^{O}[3]$, it follows that

$$
\Omega_{n}^{O, S O} \cong \Omega_{n-1}^{S O} \oplus \Omega_{n-2}^{O} \quad[9]
$$

We now extend the isomorphism (3.1) to pairs of spaces $(X, A)$.

Let $\left[M^{n}, B^{n-1} ; f\right] \in \Omega_{n}^{O, S O}(X, A)$ and let $E^{n}$ be the orientation double covering of $M^{n}$. Then there is an equivariant differentiable map $g:\left(T, E^{n}\right)$ $\rightarrow\left(A, S^{N}\right)(N$ large $)$ which is $t$-regular on $S^{N-1}$ and such that $g\left(B^{n-1}\right)=$ $(0, \ldots, 0,1)$ and $g\left(-B^{n-1}\right)=(0, \ldots, 0,-1)$. (Here $B^{n-1} \cup-B^{n-1} \subset E^{n}$ is that part of the orientation double covering above $B^{n-1} \subset M^{n}$.) Set $N_{1}=$ $g^{-1}\left(S^{N-1}\right)$. Then $N^{n-1}=N_{1}^{n-1} / T$ is an oriented submanifold of $M^{n}$. Define

$$
\Delta\left(\left[M^{n}, B^{n-1} ; f\right]\right)=\left[N^{n-1}, f^{1} \times \bar{g}^{-1}\right]
$$

where $f^{1}=f / N^{n-1}$ and $\bar{g}^{1}$ is the composition $N^{n-1} \rightarrow \bar{g}^{\bar{g}} R P(N-1) \subset R P(\infty)$ and $\bar{g}$ is the map of quotients induced by $g$. Well-known arguments [4], [12] can now be used to show that

Theorem 2. $\Delta$ is a well-defined isomorphism of degree -1 of the homology theory $\left\{\Omega_{*}^{O, S O}(X, A), f_{*}, \partial\right\}$ to the homology theor.y $\left\{\Omega_{*}^{S O}((X, A) \times R P(\infty))\right.$, $\left.(f \times \mathrm{id})_{*}, \partial^{1}\right\}$ on the category of $\mathrm{CW}$-pairs $(X, A)$.

4. Characteristic numbers. If $\gamma_{n}$ and $\gamma_{n}^{1}$ denote universal $O(n)$ - and $S O(n)$-bundles with $n$-cell as fiber and CW base space, there is an $O(n)$-bundle map $v: \gamma_{n}^{1} \rightarrow \gamma_{n}$ which is cellular on the base spaces. By using the mapping cylinder of the map of base spaces $\bar{v}: B S O(n) \rightarrow B O(n)$, one can view 
$B S O(n)$ as a subcomplex of $B O(n)$. By taking induced bundles one can view the universal $S O(n)$-bundle as the restriction of the universal $O(n)$-bundle to $B S O(n) \subset B O(n)$. Furthermore, if $\left(M^{n}, \partial M^{n}\right)$ is an $(O, S O)$-manifold, there is a unique classifying map $f:\left(M^{n}, \partial M^{n}\right) \rightarrow(B O(n), B S O(n))$. Let $f_{1}: M^{n} \rightarrow$ $B O(n)$ and $f_{2}: \partial M^{n} \rightarrow B S O(n)$ be the maps defined by $f$.

From known facts concerning the mod 2 cohomology of $B S O(n)$ and $B O(n)$ [2], it is easily shown that $H^{*}\left(B O(n), B S O(n) ; Z_{2}\right)$ is isomorphic to the ideal in $H^{*}\left(B O(n) ; Z_{2}\right)$ generated by $W_{1}$. The Stiefel-Whitney classes $w_{1}, \ldots, w_{n}$ of the $(O, S O)$-manifold $\left(M^{n}, \partial M^{n}\right)$ are defined as follows:

Let $f:\left(M^{n}, \partial M^{n}\right) \rightarrow(B O(n), B S O(n))$ be the unique classifying map given above. Define $w_{1}=f^{*}\left(W_{1}\right)$, where

$$
f^{*}: H^{1}\left(B O(n), B S O(n) ; Z_{2}\right) \rightarrow H^{1}\left(M^{n}, \partial M^{n} ; Z_{2}\right)
$$

and define $w_{i}=f_{1}^{*}\left(W_{i}\right)$ for $2 \leq i \leq n$, where $f_{1}^{*}: H^{i}\left(B O(n) ; Z_{2}\right) \rightarrow H^{i}\left(M^{n} ; Z_{2}\right)$.

The Whitney numbers for an $(O, S O)$-manifold $\left(M^{n}, \partial M^{n}\right)$ are defined using the cup product pairing

$$
H^{*}\left(M^{n}, \partial M^{n} ; Z_{2}\right) \oplus H^{*}\left(M^{n} ; Z_{2}\right) \rightarrow H^{*}\left(M^{n}, \partial M^{n} ; Z_{2}\right)
$$

If $\left(i_{1}, \cdots, i_{n}\right)$ is a partition such that $i_{1}+2 i_{2}+\cdots+n i_{n}=n$, then the cup product $w_{1}^{i_{1}} \ldots . . w_{n}{ }_{n}^{n}$ is in $H^{n}\left(M^{n}, \partial M^{n} ; Z_{2}\right)$ and the Whitney number corresponding to the partition $\left(i_{1}, \cdots, i_{n}\right)$ is defined to be the Kronecker product $\left\langle w_{1}^{i} 1_{2} w_{2}^{i} \cdot \ldots \cdot w_{n}^{i}, \sigma_{n}\right\rangle$, where $\sigma_{n}{ }^{n}$ is the fundamental cycle in $H_{n}\left(M^{n}, \partial M^{n} ; Z_{2}\right)$.

Theorem 3. If $\left(M^{n}, \partial M^{n}\right)$ bords in $\Omega_{n}^{O, S O}$, then all the Whitney numbers of $\left(M^{n}, \partial M^{n}\right)$ involving $w_{1}$ are zero and all the Pontrjagin numbers of $\partial M^{n}$ are zero.

Proof. Since $\left[M^{n}, \partial M^{n}\right]=0$ in $\Omega_{n}^{O, S O}$, we have by $(2.1)$ that $\left[\partial M^{n}\right]=0$ in $\Omega_{n-1}^{S O}$. Hence all the Pontrjagin numbers of $\partial M^{n}$ are zero [11].

Since $\left(M^{n}, \partial M^{n}\right)$ bords there is a manifold $W^{n+1}$ such that $M^{n} \subset \partial W^{n+1}$ and $\partial W^{n+1}$ - int $M^{n}$ is oriented in such a way that it induces the given orientation on $M^{n}$. Let $w_{1}, \cdots, w_{n}$ be the Stiefel-Whitney classes of $\left(M^{n}, \partial M^{n}\right)$, and let $w_{1}^{\prime}, \cdots, w_{n}^{\prime}, w_{n+1}^{\prime}$ be the Stiefel-Whitney classes of $\left(W^{n+1}, \partial W^{n+1}-\right.$ int $\left.M^{n}\right)$. The inclusion map

$$
j \circ i:\left(M^{n}, \partial M^{n}\right) \stackrel{i}{\longrightarrow}\left(\partial W^{n+1}, \partial W^{n+1}-\text { int } M^{n}\right) \stackrel{j}{\longrightarrow}\left(W^{n+1}, \partial W^{n+1}-\text { int } M^{n}\right)
$$


has $(j \circ i)^{*}\left(w_{k}^{\prime}\right)=w_{k}$ for $1 \leq k \leq n$. Let $\sigma_{n} \in H_{n}\left(M^{n}, \partial M^{n} ; Z_{2}\right)$ and $\sigma_{n+1}^{1} \epsilon$ $H_{n+1}\left(W^{n+1}, \partial W^{n+1} ; Z_{2}\right)$ be the fundamental cycles. From the exact sequence of the triple $\left(W^{n+1}, \partial W^{n+1}, \partial W^{n+1}-\right.$ int $\left.M^{n}\right)$, it follows that $i_{*}^{-1} \partial\left(\sigma_{n+1}^{1}\right)=\sigma_{n}$. Then

$$
\begin{aligned}
& \left.\left\langle w_{1}^{i}{ }^{1} \cdot \ldots \cdot w_{n}^{i}{ }_{n}, \sigma_{n}\right\rangle=\left\langle i^{*}{ }^{*}\left(w_{1}^{\prime}{ }^{i} 1\right) \cdot \ldots \cdot w_{n}^{{ }^{i} n}\right), i_{*}^{-1} \partial\left(\sigma_{n+1}^{1}\right)\right\rangle \\
& =\left\langle\delta i^{*-1} i^{*}{ }^{* *}\left(w_{1}^{i^{1}} \cdot \ldots \cdot w_{n}^{\prime i}{ }^{i}\right), \sigma_{n+1}^{1}\right\rangle \\
& =\left\langle\delta j^{*}\left(w_{1}^{i} 1 \cdot \ldots \cdot w_{n}^{i_{n}}\right), \sigma_{n+1}^{1}\right\rangle \\
& =0, \quad \text { since } \delta j^{*}=0 \text {. }
\end{aligned}
$$

Hence all the Whitney numbers of $\left(N^{n}, \partial M^{n}\right)$ are zero, and the proof is complete.

The following is an immediate consequence of this theorem.

Corollary 1. If $\left(M_{1}^{n}, \partial M_{1}^{n}\right)$ is bordant to $\left(M_{2}^{n}, \partial M_{2}^{n}\right)$, then the Whitney numbers of $\left(M_{1}^{n}, \partial M_{1}^{n}\right)$ and $\left(M_{2}^{n}, \partial M_{2}^{n}\right)$ involving $W_{1}$ are equal and the Pontrjagin numbers of $\partial M_{1}^{n}$ and $\partial M_{2}^{n}$ are equal.

Note that if a class in $\Omega_{n}^{O, S O}$ is represented by a manifold without boundary, then the Whitney numbers turn out to be the ordinary Whitney numbers.

Theorem 4. If $\left(M^{n}, \partial M^{n}\right)$ is an $(O, S O)$-manifold such that all the Whitney numbers of $\left(M^{n}, \partial M^{n}\right)$ involving $w_{1}$ are zero and all the Pontrjagin numbers of $\partial M^{n}$ are zero, then $\left[M^{n}, \partial M^{n}\right]=0$ in $\Omega_{n}^{O, S O}$.

Proof. Recall the exact sequence $\Omega_{n}^{O} \rightarrow^{j_{*}} \Omega_{n}^{O, S O} \rightarrow^{\partial} \Omega_{n-1}^{S O}$. Since $\partial M^{n}$ is a boundary, all of its Whitney numbers are zero. Hence $\left[\partial M^{n}\right]=0$ in $\Omega_{n-1}^{S O}$. That is, $\left.\partial\left(M^{n}, \partial M^{n}\right]\right)=\left[\partial M^{n}\right]=0$. By exactness there exists $N^{n}$ such that $j_{*}\left(\left[N^{n}\right]_{2}\right)=\left[N^{n}, \varnothing\right]=\left[M^{n}, \partial M^{n}\right]$. By Corollary 1 the Whitney numbers of $\left(N^{n}, \varnothing\right)$ involving $w_{1}$ are all zero. But the Whitney numbers of $\left(N^{n}, \varnothing\right)$ are the same as those of $N^{n}$. Hence the class $\left[N^{n}\right]_{2}$ in $\Omega_{n}^{O}$ contains an oriented manifold; that is, $\left[N^{n}\right]_{2}$ is in im $r=\operatorname{ker} j_{*}$. It follows that $j_{*}\left(\left[N^{n}\right]_{2}\right)=0=\left[M^{n}, \partial M^{n}\right]$ and the theorem is proven.

The following corollary is immediate.

Corollary 2. $\left(M_{1}^{n}, \partial M^{n}\right)$ is bordant to $\left(M_{2}^{n}, \partial M_{2}^{n}\right)$ if and only if the Whitney numbers of $\left(M_{1}^{n}, \partial M_{1}^{n}\right)$ involving $w_{1}$ are equal to those of $\left(M_{2}^{n}, \partial M_{2}^{n}\right)$ and the 
Pontrjagin numbers of $\partial M_{1}^{n}$ equal those of $\partial M_{2}^{n}$.

5. Concluding remarks. If the Thom spectrum $M S O$ is viewed as a subspectrum of $M O$, then, as in [5], we may consider the quotient spectrum $M O / M S O$. The techniques of $[4, \S I I]$ can be used to prove that there is an isomorphism $\Omega_{*}^{O, S O}(X, A) \rightarrow \Pi_{*}((X, A) \wedge M O / M S O)$ of homology theories over the category of $\mathrm{CW}$ pairs $(X, A)$.

\section{REFERENCES}

1. M. F. Atiyah, Bordism and cobordism, Proc. Cambridge Philos. Soc. 57 (1961), 200-208. MR 23 \#A4150.

2. A. Bord. La cohomologie mod 2 de certains espaces homogènes, Comment. Math. Helv. 27 (1953), 165-197.

3. R. O. Burdick, Manifolds fibered over the circle, Thesis, University of Virginia, Charlottesville, Va., 1966.

4. P. E. Conner and E. E. Floyd, Differentiable periodic maps, Ergebnisse der Mathematik und ihrer Grenzgebiete, N. F., Band 33, Academic Press, New York; Springer-Verlag, Berlin, 1964. MR 31 \#750.

5. - The relation of cobordism to K-theories, Lecture Notes in Math., no. 28, Springer-Verlag, Berlin and New York, 1966. MR 35 \#7344.

6. J. W. Milnor, Differentiable structures on homotopy spheres, Princeton, 1959. (mimeographed notes).

7. - On the cobordism ring $\Omega^{*}$ and a complex analogue. I, Amer. J. Math. 82 (1960), 505-521. MR 22 \#9975.

8. V. A. Rohlin, Intrinsic homologies, Dokl. Akad. Nauk SSSR 89 (1953), 789792. (Russian) MR 15, 53.

9. R. E. Stong, Notes on cobordism theory, Princeton Univ. Press, Princeton, N. J., 1968. MR 40 \#2108.

10. R. Thom, Quelques propriétés globales des variétés différentiables, Comment. Math. Helv. 28 (1954), 17-86. MR 15, 890.

11. C. T. C. Wall, Determination of the cobordism ring, Ann. of Math. (2) 72 (1960), 292-311. MR 22 \#11403.

12. E. H. Spanier, Algebraic topology, McGraw-Hill, New York, 1966. MR 35 $\# 1007$.

DEPARTMENT OF MATHEMATICS, UNIVERSITY OF ALABAMA, UNIVERSITY, ALABAMA 35486 